ScIDice
International Journal of Dentistry and Oral Science (IJDOS)

ISSN: $2377-8075$

\title{
Socket Preservation - A Case Report
}

Kushal Shah, Poonam Rai ${ }^{1}$, Devanand Shetty ${ }^{2}$, Bhargavi Sheth ${ }^{3}$

${ }^{1}$ Post Graduate Student, Department of Periodontics and Oral Implantology, D Y Patil School of Dentistry, Navi Mumbai, India.

${ }^{1}$ Professor, Department of Periodontics and Oral Implantology, D Y Patil School of Dentistry, Navi Mumbai, India.

${ }^{2}$ Professor \& Head of Department, Department of Periodontics and Oral Implantology, D Y Patil School of Dentistry, Navi Mumbai, India.

\section{Abstract}

Post the extraction of a tooth, there is alveolar bone resorption in that area, which leads to further complications in terms of prosthetic and surgical situations when the tooth needs to be replaced. To serve this problem of dimensional reduction of alveolar bone around the missing tooth, socket preservation offers an ideal solution. This case report discusses the extraction of a root-canal treated right mandibular molar which was extracted atraumatically by hemisectioning the tooth and preserving the inter-radicular bone followed by grafting the socket with demineralized freeze dried bone allograft and PerioCol membrane. After a healing period of 3-months a CBCT scan was done to evaluate the regeneration of the grafted site and bone fill. This brings us to a conclusion that grafting the socket post-extraction, helps reduce the dimensional changes for better prosthetic rehabilitation.

Keywords: Socket Preservation; Demineralized Freeze Dried Bone Allograft; Periocol Membrane.

\section{Introduction}

Each time a tooth is lost due to caries or any other cause; there are morphological and dimensional changes in the alveolar bone surrounding that region. These changes are observed both in the vertical and horizontal directions [1]. Bone remodelling takes place in the initial 3-6 months after the tooth is extracted [2]. This is a critical phase wherein maximum dimensional changes are expected. Therefore, preservation of the alveolar socket post extracting the tooth plays an important role in preventing the dimensional changes. This further impacts the placement of implant in that region without compromising the implant dimensions hence resulting in long-term success of the implant. If the socket is compressed after tooth extraction, there may be severe bone defects near the socket, which will hamper the further prosthetic rehabilitation of that area.

\section{Case Report}

A 23-year-old male patient reported to the department with a complaint of a fractured tooth in the lower right back region of the jaw. The patient was systemically healthy. He gave a history of root canal treatment in relation to the same tooth. On clinical examination it was found that there was insufficient crown height (FIG 1). The patient was advised a CBCT scan from another department to evaluate the cause of pain, which revealed a fracture line along with some periapical pathology in relation to tooth number 46 (FIG 2a,2b). The treatment plan comprising of an atraumatic tooth extraction by hemisectioning the tooth, followed by socket preservation with demineralized freeze dried bone allograft and PerioCol membrane, was discussed with the patient. An informed consent was obtained from the patient followed by scaling and root planing 2 weeks prior to the procedure. On the day of the procedure local anesthesia ( $2 \%$ lignocaine with 1:80,000 adrenaline) was administered to the patient. A full thickness mucoperiosteal flap was reflected beyond the mucogingival junction and the tooth was hemisectioned with the help of an airotor and a straight diamond bur (FIG 3). A radiograph was taken to evaluate whether or not the vertical groove of hemisection has reached the furcation of the tooth (FIG 4). Once the desired depth of hemisection was attained, Periotomes were used for extracting the tooth atraumatically while simultaneously preserving the inter-radicular bone. Curettage of the extraction socket was done using a spoon excavator and the socket was rinsed with

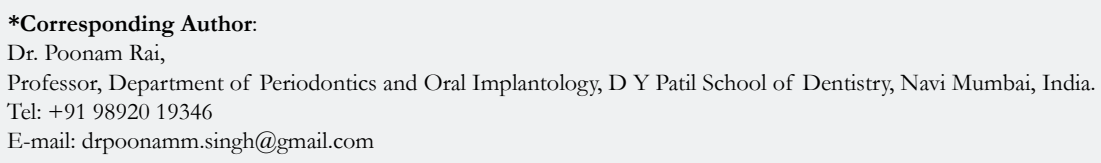

Copyright: Poonam Rai ${ }^{\circ}$ 2022. This is an open-access article distributed under the terms of the Creative Commons Attribution License, which permits unrestricted use, distribution and reproduction in any medium, provided the original author and source are credited. 
sterile saline(FIG 5). PerioCol membrane was adapted around the socket after which demineralized freeze dried bone allograft (500-1000 micron particle size) was used to graft the socket upto an extent such that the membrane would not collapse into the socket (FIG 6). The flap was then coronally advanced and sutured using interrupted sutures to attain maximum closure (FIG 7). The entire procedure was performed under antibiotic coverage (Amoxicillin 500mg) post-operative medication comprising of Cap. Amox 500 TDS for 5 days, Metronidazole $400 \mathrm{mg}$ TDS for 5 days and Ibuprofen $400 \mathrm{mg}$ BID for 3 days were prescribed. Suture removal was done after 2 weeks. After 1 month the patient was recalled and evaluated for healing which was satisfactory. Patient was recalled after 3 months for clinical and CBCT scan to evaluate the bone fill. On CBCT evaluation it was seen that there was minimum amount of radiolucency at the grafted site (FIG 9a, 9b). Clinical examination of the surgical site showed complete soft tissue healing (FIG 8).

\section{Discussion}

Studies have quoted the use of guided bone regeneration techniques in socket preservations gives better results, especially when a membrane is used [3]. In this case a socket preservation was done along with raising a full thickness mucoperiosteal flap, there are certain known disadvantaged of raising a flap during socket preservation as there is difficulty in attaining primary closure along with increased tension in the flap and reduction of vestibular depth. To overcome these drawbacks newer techniques are used which are more conservative as a flap reflection is not required $[4,5]$. The grafting material should be biocompatible, osteoconductive and osteoinductive [6]. Xenografts and alloplasts with or without a membrane have shown adequate results for socket preservation. Few studies have also used a combination of allografts; however, there are negligible differences in changes to alveolar ridge dimensions when comparing DFDBA to combination allografts [7]. DFDBA has been extensively used in periodontal treatment and has proven to be safe, and it induces the formation of new bone. DFDBA is both osteoconductive and osteoinductive in nature. Use of DFDBA has been used in several animal studies and has proven that it could stimulate the formation of new bone by osteoinduction. DFDBA also acts as a scaffold for osteoconduction [8]. Among all the available membranes, collagen membrane was preferred as it has a high biocompatibility and haemostatic activity that facilitates clot formation and stabilizes the wound. Collagen has a high chemotactic function for fibroblasts. This promotes cell migration, and primary wound coverage [9]. In this case the ridge dimensions were preserved
Figure 1. Pre-Operative Clinical Picture.

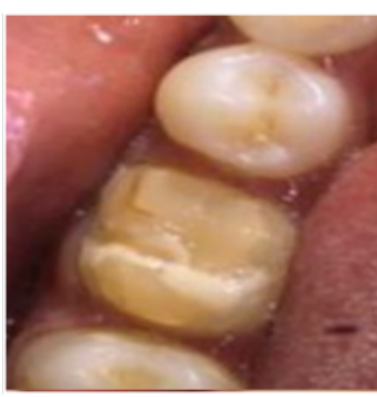

Figure 4. Radiograph After Hemisection.

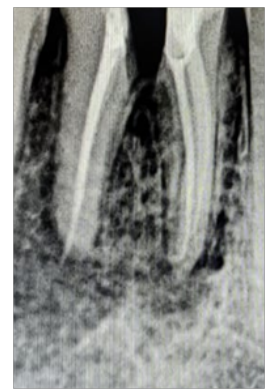

Figure 7. Interrupted Sutures Placed.

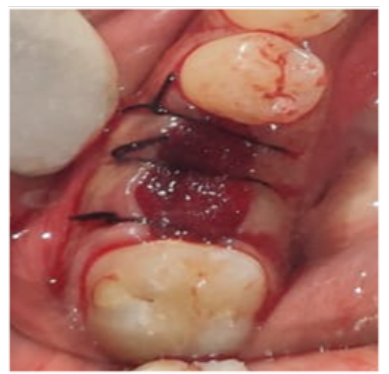

Figure 2A,2B. Pre-Operative Cbct Scan.
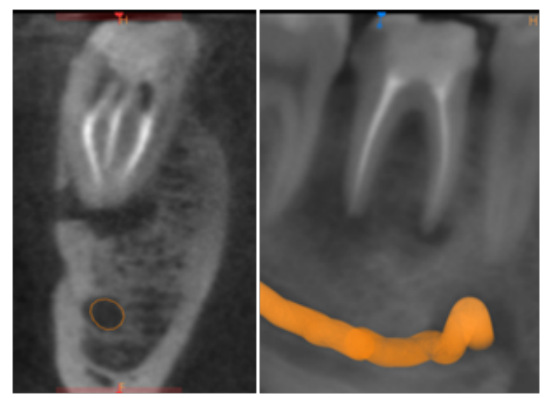

Figure 3. Hemisection Performed After Reflecting Full Thickness Mucoperiosteal Flap.

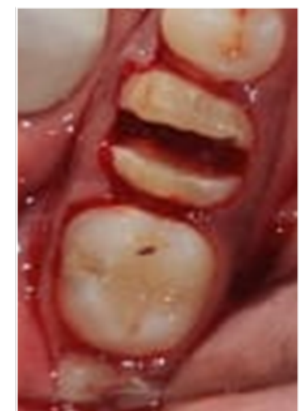

Figure 6. DFDBA Graft And Periocol Membrane Placed.

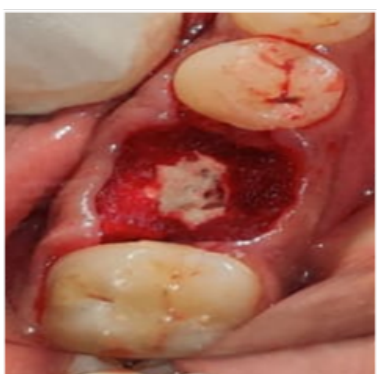

Figure 9A,9B: 3 Months Follow Up CBCT Scan.
Figure 8. 3 Months Follow Up Clinical Picture.

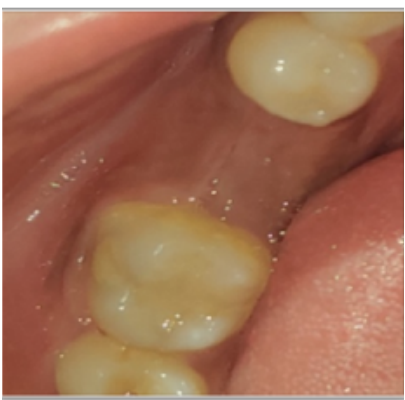

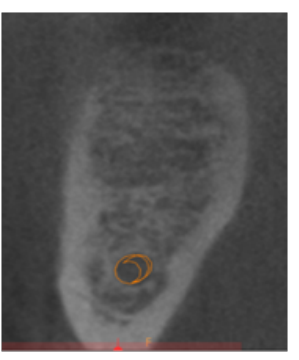

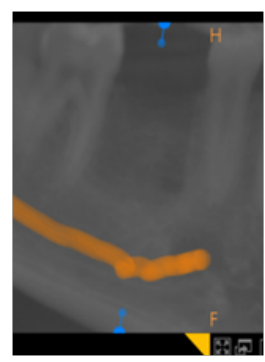


because of the use of DFDBA and PerioCol membrane for a better outcome.

\section{Conclusion}

Socket preservation done with the help of guided bone regeneration technique can minimize bone resorption and aid in further placement of implants.

\section{References}

[1]. Schropp L, Kostopoulos L, Wenzel A. Bone healing following immediate versus delayed placement of titanium implants into extraction sockets: a prospective clinical study. Int J Oral Maxillofac Implants. 2003 MarApr;18(2):189-99.Pubmed PMID: 12705296.

[2]. Kanwar S, Shetty A, Shetty D, Wadkar P. Socket preservation and reconstruction: A case report with follow up of 9 months. Int. J. Appl. Dent. Sci.2021.

[3]. Vignoletti F, Matesanz P, Rodrigo D, Figuero E, Martin C, Sanz M. Surgical protocols for ridge preservation after tooth extraction. A systematic re- view. Clin Oral Implants Res. 2012 Feb;23 Suppl 5:22-38.Pubmed PMID: 22211304.

[4]. Sclar AG. Preserving alveolar ridge anatomy following tooth removal in conjunction with immediate implant placement. The Bio-Col technique. Atlas Oral Maxillofac Surg Clin North Am. 1999 Sep;7(2):39-59.Pubmed PMID: 11905323.

[5]. Landsberg CJ, Bichacho N. A modified surgical/prosthetic approach for optimal single implant supported crown. Part I--The socket seal surgery. Pract Periodontics Aesthet Dent. 1994 Mar;6(2):11-7.Pubmed PMID: 7670061.

[6]. Yip I, Ma L, Mattheos N, Dard M, Lang NP. Defect healing with various bone substitutes. Clin. Oral Implants Res. 2015 May;26(5):606-14.

[7]. Horowitz R, Holtzclaw D, Rosen PS. A review on alveolar ridge preservation following tooth extraction. J. Evid. Based Dent. Pract. 2012 Sep 1;12(3):149-60.

[8]. Kukreja BJ, Dodwad V, Kukreja P, Ahuja S, Mehra P. A comparative evaluation of platelet-rich plasma in combination with demineralized freeze-dried bone allograft and DFDBA alone in the treatment of periodontal intrabony defects: A clinicoradiographic study. J Indian Soc Periodontol. 2014 Sep;18(5):618-23.Pubmed PMID: 25425824.

[9]. Zubillaga G, Von Hagen S, Simon BI, Deasy MJ. Changes in alveolar bone height and width following post-extraction ridge augmentation using a fixed bioabsorbable membrane and demineralized freeze-dried bone osteoinductive graft. J Periodontol. 2003 Jul;74(7):965-75.Pubmed PMID: 12931758. 